\title{
Sobre políticas locales de seguridad para jóvenes
}

\author{
Augusto Caccia-Bava*
}

\begin{abstract}
Resumen
Los jóvenes, reconocidos como categoría social en movimiento, tienen que ser referencias de las políticas públicas locales sobre seguridad. Para ello, se debe concebir la seguridad como expresión de la movilización de grupos y de comunidades activas, en el seno de la sociedad civil, como también el reconocimiento de los jóvenes a partir de sus capacidades. Investigaciones y discusiones teóricas realizadas en el ámbito de institutos brasileños y del Observatorio Catalán de la Juventud iluminan este nuevo paradigma.
\end{abstract}

Palabras clave: políticas públicas, seguridad urbana, juventud, formación cultural.

\begin{abstract}
The young, recognized as a social group in motion, have to be references of the local public policies on security. For that purpose, security should be conceived as the expression of active groups' and communities' movements, in the heart of civil society, as well as the recognition of the young's capabilities. Research and theoretical discussions carried out in Brazilian institutes and in the Catalonian Observatory of the Youth shed light on this new paradigm.
\end{abstract}

Key words: public policies, city security, youth, cultural education.

* Brasileño, Profesor Asistente Doctor del Departamento de Sociología de la Faculdade de Ciências e Letras -UNESP- Campus de Araraquara, São Paulo. Vice-coordinador del programa de Postgrado en Sociología, de la misma universidad. Presidente del Centro Brasileño de la Infancia y de la Juventud y miembro regular del Foro Latinoamericano para Seguridad Urbana y Democracia, México-DF. Autor y coeditor de Jovens na América Latina, Editora Escrituras/Cebrij, 2004; miembro del Consejo de Redacción y autor de ensayos de la revista Estudos de Sociología del Departamento de Sociología y del Programa de Postgrado en Sociología de la Unesp. E-mail: augusto@flcar.unesp.br 
Las políticas sobre seguridad para los jóvenes, como aspecto central de políticas públicas locales para esta categoría social, es el tema del presente texto. Como veremos, la seguridad, entendida en su dimensión urbana preventiva, ya viene siendo objeto de reflexión metódica en América Latina desde 2000, fecha en que se inician también investigaciones acerca del perfil de la violencia en Brasil.

Algunos autores latinoamericanos nos proporcionan sólidos pilares a la hora de entablar la discusión sobre políticas locales para la juventud. Iniciamos el debate con Juan Carlos Rubinstein, jurista y también doctor en Ciencias Sociales, y más específicamente con las propuestas defendidas en su libro Crisis de la sociedad civil: neofeudalización -posfordismo.

Rubinstein es uno de los teóricos que reconoce la existencia de transformaciones objetivas en las relaciones sociales, lo que conlleva el "renacer localista". Para él, se trata de "una suerte de regresión colectiva, tendientes a fijar [...] un punto de referencia que les permita [a los grupos] objetivar su situación en un espacio y en un tiempo, que se ha movilizado y cambiado aceleradamente. Y tal movilidad es consecuencia de las debilidades de la sociedad civil y no una expresión de su fuerza" (Rubinstein, 2002, 169).

Rubinstein menciona igualmente la presencia de un marco igualitario que se construye en el seno de la sociedad civil. Sin embargo, no se trata de producir igualdad de hecho entre los grupos, como resultado de la superación de contradicciones antagónicas, sino de una probabilidad de existencia, a través de la movilidad. Por lo tanto, podemos afirmar que los estudios sobre manifestaciones identitarias desarrolladas por diferentes grupos se encaminan hacia alternativas al orden constituido, dentro de ese mismo orden. ¿Cómo? Ahondando y radicalizando el análisis para comprender la "acción política, ya que, al contemplarse sus intereses [de los grupos], se atiende con más precisión a los 
contenidos de la lucha y las interrelaciones de clase" (Rubinstein, 2002, 171, 178).

Pablo González Casanova, sociólogo mexicano, va más allá al indicar aspectos importantes de las formas cotidianas de existencia, situándolas en el presente proceso histórico: "Si el neocapitalismo de Estado asistencialista acabó el concepto-fuerza de los proletarios de todos los países capaces de unirse y lo hizo mediante la estratificación de la clase trabajadora, el capitalismo transnacional, en su etapa de deglobalización, acentúa las diferencias de los trabajadores mediante estructuras focalizadas o localizadas que alteran aún más el pensar y el hacer de las políticas de interés general, de por sí limitadas y eliminan buena parte de los alcanzados por los trabajadores organizados (González Casanova, 2002, P. 281-282).

La eliminación de las referencias históricas de un pasado de lucha, tradición y principio de autoridad, principios constituidos por antagonismos entre grupos de diferentes clases, acaba por producir lo que se conoce por "opciones de políticas focalizadas" que "alteran categorías enteras, como las de clase o nación". Por otra parte, estas "desintegran las categorías sociales y políticas", transformándolas en "intereses particulares" que crean obstáculos al esfuerzo de "concebir-expresar-actuar de la $<<$ clase $>>$ o de la nación". Naturalmente, esta política e ideología dominantes se tornan posibles porque resultan consecuencias de estrategias formuladas en la lógica del capital financiero cuya propuesta es ensanchar los espacios de libertad para su realización, es decir, se trata de crear todas las posibilidades de libertad para la acumulación privada (González Casanova, 2002, p. 282).

Ramón Casanova, sociólogo venezolano, se sitúa en un horizonte teórico similar al del sociólogo mexicano. Casanova integra un colectivo de discusión que organizó un seminario, en 2004, sobre lo que se denominó "pensamiento que guía la acción pública, en el actual proceso político bolivariano". Se trata de "explorar 
una interpretación de las claves intelectuales que están detrás del diseño de la política social". Para él, la crítica frente a las políticas públicas tiene que considerar los distintos movimientos políticos presentes en cada coyuntura y, en particular, los proyectos que se presentan como alternativos y que señalan "una reestructuración de los principios que definen y regulan la institucionalidad del Estado" (Casanova, 2004, fls. 1).

Según este crítico del pensamiento dominante en nuestros días, en particular del pensamiento demócrata social que defiende una gobernabilidad abstracta, es posible concebir el "experimento de aceleración histórica heterodoxo que compite abiertamente con el programa neoliberal" en territorios de discusión política, institucionales o no. Las experiencias se convierten en "el laboratorio para el debate en torno a las vías posibles de desarrollo alternativo al modo predominante de la globalización" (Casanova, 2004, fls. 03).

La crítica contra las políticas focales o localizadas para la juventud exige, en esta perspectiva que también defendemos, que se cuestionen sus fundamentos y sus fuentes de inspiración. Ello, desde un concepto de Estado y de acción política que resulte la extensión de una "una filosofía política de la desigualdad", hoy encubiertas por un atributo en boga: lo natural. La desigualdad natural sería la razón de la intervención pública dentro de "lo posible", sin que se pretenda modificar las estructuras reproductoras de la iniquidad.

En otro ángulo, se sitúa el sociólogo al intentar recuperar "valores republicanos esenciales de liberté, égalité y fraternité" (Casanova, 2004, fls. 08) ${ }^{1}$.

1 Países como Venezuela aportan nuevas experiencias colectivas para enriquecer las investigaciones sobre temáticas locales, lo que, según el sociólogo venezolano Ramón Casanova, refleja igualmente la falta de capacidad política, más que la ausencia de recursos financieros, a la hora de enfrentar los problemas sociales de las comunidades. 


\section{La narco-economía latinoamericana}

En un ensayo bien elaborado, Hardinghaus (1995) expone las ramificaciones de la narco-economía y sus relaciones con la limitada capacidad del Estado para detener esa dimensión reproductora del capital. Tal dimensión cabe ser considerada como una de las fuentes de iniquidad social en Brasil. Esta economía ilícita revela también aspectos contradictorios sobre seguridad urbana, temática que afecta a los jóvenes en nuestro país, y, en mayor o menor medida, al resto de América Latina. Uno de estos aspectos es la educación que puede ser alternativa contra la violencia urbana.

La ingenuidad de la educación para el uso de drogas se produce por la abstracción de conceptos como drogas puras o impuras, lo que presupone una caracterización en la lógica del mercado. El mismo autor subraya: "se Lanza al mercado (primera mitad de los años 80) el crack, la novedad más exitosa de los últimos veinte años: cocaína para fumar". A esta droga, se le suman las drogas sintéticas, que llegan con las músicas de las discoteca: "a cada música su propia droga" o a través de las "tecnopíldoras", diseminadas en los locales de difusión de la música tecno (Hardinghaus, 1995, fls. 03).

¿Resultaría lícito cuestionar, pues, cuál sería la política focal adecuada para la juventud, en los diferentes estados del País, frente a esa nueva ola narcótica y mercantilista? ¿Sería posible identificar en las denominadas políticas locales para la juventud referencias para enfrentar el proceso de difusión de la narco-economía? Si la respuesta resulta negativa, tendremos que indagar si es posible identificar otras políticas públicas locales, distintas de las existentes, que puedan combatir de raíz este proceso destructivo que atañe esencialmente a las nuevas generaciones.

Huelga decir que los llamados estupefacientes fueron diseminados por el mundo en oleadas: la asiática, de opio, durante las dos primeras décadas del siglo XX; la de marihuana y hachís, 
en los Estados Unidos, durante los años 60 y 70. La de cocaína, también en los EEUU, en los años 80 y, más recientemente, las drogas sintéticas -designer drugs- mayoritariamente consumidas en California (Hardinghaus, 1995, idem).

La drogadicción posee márgenes definidos en el mercado mundial. Lo que también conlleva concepciones ecológicas de pureza: "los movimientos ecologistas y biológicos también han tenido repercusiones en la oferta de estupefacientes. Los oferentes de drogas naturales se defienden de las drogas $<<$ diseñadas $>>$, aseguran los mercados, se diversifican". Por este motivo, Colombia, uno de los países citados por el autor, se convirtió en un productor diversificado de drogas: "Colombia fue, en principio, un productor de marihuana, se convirtió en el mayor procesador de cocaína y ahora cultiva amapola...para la producción de heroína" (Hardinghaus, 1995, fls. 04).

El autor presenta una base para posteriores investigaciones sobre tal proceso, base que puede convertirse en referencia para colectar datos en una investigación de mayor magnitud que la presente:

Se pueden lograr aproximaciones a la realidad desde el área de la investigación policial (informes de acusados y testigos), de la asistencia sanitaria y terapéutica (informes sobre comportamiento, frecuencia y tasa del consumo, adictos registrados, hospitalizaciones, fallecidos por uso de estupefacientes), de la vigilancia militar (fotos aéreas y de satélites de zonas de cultivo y laboratorios), de la represión aduanera del contrabando (decomisos), de investigaciones de campo realizadas por científicos, de entrevistas de periodistas especializados con traficantes, productores o transportistas, de los movimientos de fondos en las áreas de flujo intensivo de narcodinero, de las estadísticas del sistema financiero mundial y de los bancos centrales nacionales (Hardinghaus, 1995, fls. 05).

Finalmente, el hecho de formular políticas públicas para 
proteger a los jóvenes amenazados por la narco-economía podría constituirse en una referencia para todas las políticas locales destinadas a la juventud. Estas deberían estructurarse a base de la identificación de la cadena productiva, pero también de la cadena reproductiva de la violencia que, como afirma Ianni (2004), se convirtió, más que en un fenómeno estructural, en una mediación de la estructura productiva. Para él, la violencia:

“Nace como técnica de poder, se ejerce también como un modo de preservar, ampliar o conquistar la propiedad y adquiere nuevos matices psicológicos sorprendentes en lo que respecta a los agentes y a las víctimas. Entra como elemento importante de la política de la cultura..." (IANNI, 2004, p. 169).

Por estos motivos, entre otros, resulta cada vez más pertinente desarrollar investigaciones en los ámbitos regional y local. Algunas ciudades son blancos preferenciales de las personas que estructuran el comercio de drogas. Como afirma Hardinghaus, "el dinero es el acompañante fijo de la droga, su amante furtivo (y tenebroso). Es el combustible de todo el proceso de producción y venta, recorre el camino de ida hacia el consumidor final, y también el de regreso, del distribuidor al productor" (Hardinghaus, 1995, fls. 10).

En el seno de la Secretaría Especial de Derechos Humanos, creada en los primordios del gobierno Lula, se plantearon desafíos nacionales que defendiesen la integridad de la vida de los brasileños y de extranjeros que aquí residen. Estos temas deben y pueden tratarse en el dominio de los derechos humanos y de la seguridad pública, con el fin de ampliar los horizontes para una mejor comprensión de los mismos². Las principales temáticas se-

2 En la página principal de la Internet del Ministerio de Justicia brasileño se pueden recoger informaciones sobre el órgano responsable por combatir el trabajo esclavo. Se trata de la Comissão Nacional para a Erradicação do Trabalho Escravo - CONATRAE, que tiene por función primordial, monitorear la puesta en práctica del Plano Nacional por la Erradicación del Trabajo Esclavo. El Plano, que contiene 76 acciones, (Cf. www. mj.gov.br), surgió el 11 de marzo de 2003. 
rían: I. La presencia del trabajo esclavo en latifundios agrarios. II. La existencia de más de cien rutas de prostitución en el territorio nacional, algunas con nexos internacionales. En el espacio local, se puede observar esta segunda práctica que hiere los derechos humanos y promueve inseguridad pública tanto en los espacios urbanos concretos como en los espacios virtuales. Como se relaciona con el tema que desarrollamos, indico algunos de sus territorios:

- Redes de entretenimiento (shopping center), discotecas, bares, restaurantes, moteles, bares de playa, salones de danza, casas de shows, locales destinados a las escuelas de samba, prostíbulos, casas de masajes....

- $\quad$ Redes del mercado de moda (fashion): agencias de modelos (fotográficos, de videos y películas).

- Redes de agencias matrimoniales (entre los modos de inserción en las redes del tráfico; este es el que mayores problemas plantea a la hora de caracterizarlo como tal, como tráfico. Esto, porque conlleva aspectos amorosos y afectivos inherentes a la relación interpersonal).

- Red de tele-sexo: anuncios en periódicos, internet y televisión (circuito interno).

- Red de la industria del turismo: agencias de viajes, hoteles, balnearios/resorts, taxistas, transporte turístico. (PESTRAF, 2002).

Encontramos los primeros registros de violencia a nivel nacional con incidencia seria en los jóvenes, en la página electrónica de la UNESCO-Brasil, que contiene referencias de la investigación que analizó diferentes situaciones de violencia contra jóvenes brasileños.

A principios de 2003, se tornaron públicos los primeros resultados exitosos del llamado Mapa de la Violencia. La cuarta edición 
del mismo se destinó al estudio de los jóvenes en situación de violencia. Este documento indica que $11 \%$ de todos los homicidios mundiales se llevan a cabo en Brasil. Por ello, ocupa el quinto lugar en la lista mundial sobre jóvenes que fallecen por causas externas y violentas. Colombia, Islas Vírgenes, El Salvador y Venezuela ocupan los primeros lugares de la lista. Sin embargo, la aparición a la cabeza de otros tres países latinoamericanos y de otro territorio, antigua colonia británica, de ninguna manera nos deja la conciencia tranquila.

Para el sociólogo y coordinador de la investigación, Julio Jacobo Waiselfisz, la exclusión social es la principal responsable por la muerte de estos jóvenes. La solución sería la elaboración de políticas públicas para la juventud. La forma de exclusión a que alude Waiselfisz debe entenderse en la perspectiva de Oliveira (1998), como la faceta económica de la globalización capitalista contemporánea. Por lo tanto, esta debe ser una referencia importante, aunque remota, para la discusión sobre políticas de seguridad destinadas a los jóvenes. Por ello, la exclusión social no tiene que ser ni punto focal ni de partida, ya que se presenta en un nivel excesivamente abstracto, incluso en el ámbito de cada ciudad.

En un texto que sintetiza una vasta investigación, publicada por la revista SBPC - Ciência e Cultura, Sérgio Adorno y Nancy Cardia declararon que "no hay, en Brasil, una tradición de estudios sobre violencia, crimen ni derechos humanos ${ }^{3 \prime}$, si comparamos la producción del País con la de EE UU o Canadá. Por ello, surgieron indagaciones sobre esta temática en tres campos distintos del conocimiento: Psicología, Derecho y Ciencias Sociales. Lo que resulta más significativo, en este ejercicio, es la búsqueda de una interdisciplinariedad que intente comprender científicamente los fenómenos de la violencia y de la inseguridad pública.

3 Las informaciones sobre la naturaleza de tal investigación se encuentran en: www2. rnw.nl/rnw/es/informes/ninezjuventud/act040611_violenciabrasil.html 
Aún sin tradición, la bibliografía reunida es muy amplia, como se observa en el estudio de Adorno \& Cardia (2002).

\section{Para la reflexión crítica sobre jóvenes, juventud y movimientos en el siglo XXI}

A principios del siglo XXI, el Observatorio Catalán de la Juventud, sito en Barcelona, editó una serie periódica titulada Aportacions que nos presenta temas sobre experiencias juveniles en el territorio pacífico de esa bellísima ciudad.

Feixa (2001) introduce en su ensayo Generació@: la juventut al segle XXI una discusión sobre el concepto de tiempo de los jóvenes y sus edades, entendidas estas como estados "biográficos construidos culturalmente". El proceso de formación de esta identidad dista de ser simple ya que "las condiciones sociales se configuran a partir de la interacción entre la cultura hegemónica... y las culturas de parentesco"; estas, a su vez, oriundas de las grandes tradiciones que se encuentran en los ambientes originarios de los jóvenes. Al identificar la relación entre las culturas juveniles y el tiempo histórico, Carles Feixa Pàmpols introduce la relación entre la permanencia del pasado y el contexto en que se producen las frágiles relaciones mercantiles (Feixa, 2001, pp. 7 13).

El siglo XX se presenta como el momento histórico de la democratización de las culturas juveniles, momento en que "reformas diversas en la escuela, en el mercado del trabajo, en la familia, en el servicio militar, en las asociaciones juveniles" y su entorno "permiten que surja una nueva generación consciente de estar creando una cultura propia, distinta y diferente de la de los adultos" (Feixa, 2001, p. 27).

A base de tal afirmación, viviríamos, en los días actuales, un:

"[...] modelo híbrido de la adolescencia, ambivalente, dividido entre una creciente infantilización social -que 
se expresa a través de la dependencia económica y la ausencia de espacio de responsabilidad- y una gradual maduración intelectual -que se muestra por el acceso a las nuevas tecnologías de la comunicación... el retraso permanente para acceder al trabajo y a la independencia domiciliar... un modelo de inserción 'virtual' en la sociedad." (Feixa, 2001, p. 52).

González y otros investigadores (2003) abren el debate sobre la ocupación inmobiliaria por parte de grupos juveniles y las políticas públicas en Cataluña. El tema de la independencia domiciliar del joven se asocia al reconocimiento de movimientos juveniles de resistencia que invaden inmuebles deshabitados en Barcelona. Tal acto entró en los cauces de la legalidad por iniciativa parlamentaria, lo que permitió a los poderes locales reconocer a los movimientos juveniles como interlocutores para definir o ampliar las políticas públicas. En lo que se refiere al método, se reconoce la existencia de un "cambio cultural que conduce a la sustitución progresiva de las relaciones de producción tradicionales -centradas en el mundo del trabajo-- por otras de consumo -centradas en el tiempo libre- como un eje vertebral de las identidades personales y colectivas ". Añaden los autores: "en este sentido surge el concepto de juventud ociosa” (González, 2003, 7).

También como referencia metodológica, se afirma que el contexto presente revela dos tipos de política para la juventud: la nuclear y las periféricas. Las primeras "implican modificaciones de la trayectoria vital, [y] fundan, básicamente, políticas formativas, de trabajo y de habitación". Y las periféricas son las que aportan nuestra consabida referencia de calidad de vida (González, 2003, 9).

Paralelamente, las políticas se fortalecen, explícita o implícitamente. Explícitas son aquellas llevadas a cabo por los órganos públicos (consejos o departamentos administrativos locales) que promueven políticas para la juventud. Las implícitas se relacionan con los derechos universales como educación, habitación, 
trabajo y salud, entre otros. En Cataluña, encontramos iniciativas públicas en una dimensión que todavía no existe en Brasil. Se trata de políticas integradas que consideran "las condiciones juveniles desde todos los ámbitos posibles, en una dimensión política". Tales políticas valoran más los contenidos nucleares que los periféricos (González, 2003, 10).

Cabe establecer igualmente una diferencia de naturaleza ideológica: las políticas para los jóvenes pueden expresarse desde enfoques social-demócratas o conservadores. Subrayamos estos dos porque se encuentran presentes, en mayor o menor escala, en nuestro contexto político. El segundo es más pertinente para este estudio ya que transforma "a las personas discriminadas y no a las estructuras sociales o económicas en agentes responsables por las desigualdades". Es decir, "quienes no aceptan trabajar -aunque tengan que hacerlo en condiciones precarias- son personas ociosas y, por consiguiente, delincuentes potenciales" (González, 2003, 11).

En Cataluña, surgió la propuesta de crear una renta mínima "renta ciudadana" o "sueldo social" destinado a los jóvenes parados. Sin embargo, para los investigadores sociales, esta política contradice la lógica del sistema que presupone la presencia histórica de un ejército industrial de reserva. Este callejón encontraría una posible salida a través de los movimientos de ocupación de inmuebles, a su vez, "actores que producen políticas públicas" (González, 2003, 14).

Martínez Sanmartí (2003, p. 5 y 6) va más allá en el debate sobre cultura juvenil y género. Sus argumentos iniciales se apoyan en una fuerte premisa teórico-metodológica: "es la cultura que ayuda a separar lo que tiene y lo que carece de sentido, lo que resulta importante de lo superfluo; los jóvenes que se identifican entre sí de aquellos que se presentan como antagonistas". Al ocuparse de la relación entre género y cultura, también subraya un aspecto fundamental para el estudio de los movimientos y 
políticas destinados a los jóvenes: "el análisis de la relación entre género y cultura juvenil [pone de manifiesto] nuevas ${ }^{4}$ relaciones culturales".

Cuando analiza la subjetividad, en proceso de construcción, Martínez Sanmartí señala una mediación, en cierta medida original, dentro del contexto de la reproducción material e ideológica. Para él, la "subjetivización gradual de la identidad" se expresa en el seno de las manifestaciones juveniles, más como emoción que como sensación: "la diferencia y la novedad que presenta el consumo moderno consiste en que su preocupación ya no es por despertar sensaciones sino emociones. Esto se debe a que el capital, a través de relaciones mercantiles, intentó llegar hasta un "consumidor adolescente", con capacidad para emocionarse. Por ello, afirma el autor de manera pertinente: "la cultura juvenil no es uniforme, ni evidente en sí misma". En esta misma perspectiva, añade que el género se manifiesta "en cada contexto y en cada momento histórico como masculinidad y feminidad (Martínez Sanmartí, 2003, p.7 y 9), para concluir con un sagaz argumento:

Cuando me refiero a feminidad y a masculinidad... lo hago de una manera selectiva y parcial con relación al eje que establece la diferencia ente lo que es comercial o no lo es y considera la resistencia contra la imposición de la mercantilización, como muestra del conformismo que se asocia a lo afeminado... lo importante es que, desde este punto de vista, todo lo que se considera comercial no es auténtico porque es prefabricado a partir de estrategias de marketing que impiden la auténtica creatividad artística (Martinez-Sanmartí, 2003, p.30).

El Observatorio de la Juventud de Cataluña edita otros estudios que citaremos sumariamente para integrarlos a las actuales in-

4 El término "nuevo", remite para el autor a "una licencia lingüística sospechosa". Añade Martinez- Sanmartí, (2003, p. 5 y 6) que resulta más adecuado aclarar el valor semántico del término. 
vestigaciones latinoamericanas. Entre ellos, destaca el análisis cuantitativo que incluye aspectos cualitativos sobre la situación de habitación de los jóvenes catalanes, organizado por la economista Carmem Trilla, un equipo de consultores y el Instituto de Estudios de Sociología, en 2003.

Otro estudio es el llevado a cabo por Herrera, quien, junto con otros investigadores y en 2003, debatió la cuestión de la inmigración y del trabajo intercultural en los territorios catalanes fronterizos.

Al año siguiente, Pau Miret i Gramundi se planteó en su estudio la emancipación domiciliar de los jóvenes catalanes mediante la actividad laboral. En este mismo trabajo, analiza el neorruralismo, movimiento que se propone buscar alternativas frente a la existencia de la agitada vida urbana, contaminada y en cierto sentido, despiadada.

Módenes Cabrerizo \& Recaño Valverde (2003) analizaron el concepto de territorio y el complejo proceso de movilidad migratoria de los jóvenes en Cataluña.

En 2004, y bajo la dirección de Bernat Albaigés i Blasi, se realizó un estudio sobre la crisis del trabajo y la necesidad urgente de crear nuevas normas de subjetividad laboral para los jóvenes.

Otro original estudio fue el llevado a cabo por Serracant Melendres (2005), autor que analizó la relación entre el capital y las actividades productivas de los jóvenes que, para Serracant, se caracterizan por la sobrecalificación.

Ya a finales de 2005, surge la temática Jóvenes adultos y políticas de juventud en Europa. Se trata de un parecer final realizado por el programa Inter-universitario (a nivel de Master) sobre Políticas para la juventud, bajo la dirección de Juan Manuel Patón i Casas. 


\section{Premisas para la formulación de políticas públicas para jóvenes en las ciudades brasileñas}

Volvemos al presente brasileño para presentar algunas referencias metodológicas que puedan enriquecer los análisis teóricos y de campo sobre grupos juveniles localizados en un determinado territorio físico o integrados en movimientos culturales y políticos. Este trabajo es fruto de una investigación de campo que realicé junto a la Asociación de la Juventud de Ribeirão Preto (ciudad del estado de São Paulo), en 2001, detallada en Caccia-Bava (2004) y que aquí presentamos, sucintamente, como referencias:

Primera referencia: el joven tiene capacidad para ser protagonista en la formación cultural de las nuevas generaciones, tanto en las condiciones más precarias como en las más favorables, siempre y cuando se tornen portavoces de la defensa de derechos universales del niño, del adolescente, del joven o del anciano.

Segunda referencia: la formación de los jóvenes puede ser marcadamente cultural, lo que la lleva más allá de un momento mercantil, de la experiencia del ocio.

Tercera referencia: la familia es la institución en que se originan los valores presentes en el proceso de la formación cultural. Tanto la familia de origen del joven, como otras, aquellas con las que convive o que creará en el futuro. De este modo, los jóvenes que pertenecen a familias excluidas en la práctica del ejercicio de sus derechos básicos se encuentran igualmente excluidos del ámbito de la educación, en el sentido más amplio del término, institucional.

Cuarta referencia: A partir de una reflexión rigurosa sobre los derechos de la familia y tras conocer los derechos de la infancia, adolescencia y de la juventud, los jóvenes pueden comprender las dimensiones culturales y políticas de su barrio, de su ciudad y de la sociedad en que se insertan. De este ejercicio, pueden resultar manifestaciones políticas conscientes que defiendan una 
sociedad pacífica, lo que conlleva, igualmente, la defensa de sus derechos y deberes.

Quinta referencia: con relación a la maternidad, con relación a la formación escolar y con respecto a la atención familiar de los jóvenes, la experiencia juvenil ha revelado que la conciencia del deber de los mismos debe ser, predominantemente, a favor de la preservación de la integridad de la vida en los diferentes ámbitos mencionados y alejarse, por lo tanto, de los deberes dogmáticos, morales y formales, del respeto a las normas establecidas y al patrimonio físico-arquitectónicos.

Sexta referencia: cada momento de formación de los jóvenes para el trabajo debe preservar la integridad del conocimiento y de los valores aprendidos durante los años de formación cultural, familiar, política y escolar.

Séptima referencia: los procesos educativos en los niveles básico, medio y superior, deben subordinarse, en sus parámetros, a la formación cultural de los jóvenes, de manera integral, y tener por base las referencias políticas construidas a través de la convivencia pacífica, en el seno de la sociedad, con sus utopías, sobre los pilares de los derechos constituidos y por constituir, con el fin de crear una nueva civilidad.

De este modo, las políticas públicas para los jóvenes deben asentarse en las prácticas de consolidación de distintos procesos de formación cultural para las nuevas generaciones así como en la inserción de estos jóvenes, en la dinámica de construcción de sus derechos y de los derechos ajenos, de otros grupos, comunidades y colectividades. Cabe añadir, aunque suene arrogante, que las políticas públicas deben movilizar cultural y políticamente a los jóvenes para que se supere el precario asistencialismo público y filantrópico todavía vigente. ${ }^{5}$

5 Traducción realizada por la Profesora María Dolores Aybar Ramírez. Profesora Asistente Doctora del Departamento de Letras Modernas de la Faculdade de Ciências e Letras-UNESP-Campus de Araraquara, São Paulo. E-mail: lola@fclar.unesp.br 


\section{Bibliografía consultada}

Adorno, Sérgio \& Cardia, Nancy (2002). "Nota de apresentação". In: Revista da Sociedade Brasileira para o Progresso da Ciência, número 1-54. São Paulo: Imprensa Oficial do Estado, jul/ago/set., p. 20-21.

Balardini, Sérgio (2004). "As gerações jovens da Argentina". In: Caccia-Bava, A., Feixa, C. \& González, Y. (Org) Jovens na América Latina. São Paulo: Escrituras/Cebrij, p. 15-61.

Caccia-Bava, Augusto (1999). "Solidariedade, sociabilidade e ética política: temas clássicos ou contemporâneos?". In: D'Incao, M. A. (Org). Sociabilidade: Espaço e Sociedade. São Paulo: Grupo Editores, p. 277-301.

(1999). "Exclusão social na identidade brasileira". In: Praia Vermelha / Estudos de Politica e Teoria Social. Rio de Janeiro: UFRJ/Escola de Serviço Social/Programa de Pós-Graduação, Vol. I, n. 02, páginas 127- 140.

(2004). "Direitos civis dos jovens e insegurança urbana". In: Estudos de Sociologia, ano 9, n. 17, 195 páginas. Araraquara, Departamento de Sociologia/Programa de Pós-Graduação em Sociologia, $2^{\circ}$ sem., p. 41-64.

y Paiva Da Costa, Dora I. (2004). "O lugar dos jovens na história brasileira". In: Caccia-Bava, A. \& Feixa, C. \& González, Y. (Org). Jovens na América Latina. São Paulo: Escrituras/Cebrij, p.. 63-114.

Casanova, Ramón (coord.) (2004). “Conversaciones sobre política, Estado y proyecto nacional en la transición venezolana: el itinerario de las ideas políticas". In: Primera Conversación: el programa de trabajo. Documento de Trabajo, n. 3. Caracas: Fundación Escuela de Gerencia, Cuadernillos del Proyecto, 26 de julio (mimeografiado), p. 1-14.

Correa, Lélio Bentes \& Vidotti, Tárcio José (2005). “Trabalho Infantil e Direitos Humanos: homenagem a Oris de Oliveira." São Paulo: LTr.

Feixa-Pampols, Carles (2001). "Generació@: la juventut al segle XXI". In: Aportacions, No. 12. Barcelona: Observatori Catalã de la Juventut, 59 páginas.

González Casanova, Pablo (2002). “Lo particular y lo universal a fines del siglo XX". In: Nueva Sociedad, n. 180-181.Caracas: Editorial Nueva Sociedad, p.280-298.

González, Roberto et Alli (2003). "Juventut, okupació i polítiques públiques a Ca- 
talunya". In: Aportacions, No. 18. Barcelona: Observatori Catalã de la Juventut, 48 páginas.

Hardinghaus, Nicolás H. (1995). “El desarrollo de la narcoeconomía internacional". In: Nueva Sociedad, n. 138. Caracas: Editorial Nueva Sociedad. Disponível em www.nuevasociedad.org.ve, 25 páginas.

Ianni, Octávio (2004). Capitalismo, violência e terrorismo. Rio de Janeiro: Civilização Brasileira.

Martínez-Sanmartí, Roger (2003). "Cultura juvenil i gènere: premi joventut 2000". In: Aportacions, No. 21. Barcelona: Observatori Catalã de la Juventut, 45 páginas.

Manifesto das cidades. Segurança e Democracia (2006). Disponível em www. urbansecurity.org/fesu/popup_sd Consulta realizada em 06/01/2006.

Miret I Gramundi, Pau (2004). "Emacipació domiciliària, laboral i familiar dels joves a Catalunya". In: Aportacions, No. 25. Barcelona: Observatori Catalã de la Juventut.

Oliveira, Francisco (1998). Os direitos do antivalor. Pretrópolis: Vozes.

PESTRAF (2006). Pesquisa sobre tráfico de mulheres, crianças e adolescentes para fins de exploração sexual comercial. Disponível em www.cecria. org.br Consulta realizada em 05/01/2006.

Rubinstein, Juan Carlos (2002). Crisis de la sociedad civil: neofeudalização y posfordismo. Madrid: Trama Editorial.

Schaff, Adam (1999). Langage et connaissance: essais sur la philosophie du langage. Paris: Éditions Anthropos.

Souza, Janice Tirelli Pontes (1999). Reinvenções da utopia: a militância política de jovens nos anos 90. São Paulo: Hacker Editores.

Tagle, Fernando Tenório (2000). Cultura, sistema penal y criminalidad. México: UNAM/CNCT/FCE/Metropolitana.

Urteaga, Maritza \& Perez-Islás, José Antonio (2004). “Imagens juvenis do México moderno". In: Caccia-Bava, A, Feixa, C. \& González, Y. (Org). Jovens na América Latina. São Paulo: Escrituras/Cebrij, p. 183-255. 